\title{
Indicadores e índices para o gerenciamento de águas pluviais urbanas no Brasil: situação atual e oportunidades de evolução
}

\author{
Indicators and indexes for urban stormwater management \\ in Brazil: current status and opportunities for evolution
}

Camila Angélica Baum ${ }^{1}$ (D) , Joel Avruch Goldenfum ${ }^{1}$ (D)

${ }^{1}$ Universidade Federal do Rio Grande do Sul, Porto Alegre, RS, Brasil. E-mails: eng.camilabaum@gmail.com, joel@iph.ufrgs.br

\begin{abstract}
Como citar: Baum, C. A., \& Goldenfum, J. A. (2021). Indicadores e índices para o gerenciamento de águas pluviais urbanas no Brasil: situação atual e oportunidades de evolução. Revista de Gestão de Água da América Latina, 18, e21. https://doi.org/10.21168/rega.v18e21
\end{abstract}

\begin{abstract}
RESUMO: 0 gerenciamento efetivo das águas pluviais urbanas nos municípios brasileiros enfrenta impasses, como a baixa disponibilidade de dados e capacidade técnica limitada nos órgãos e entidades responsáveis. Uma alternativa para superar parcialmente esses impasses seria um adequado conjunto de indicadores. O objetivo desse trabalho é avaliar os indicadores relacionados às águas pluviais urbanas formulados para a realidade brasileira e identificar os entraves existentes e as oportunidades de evolução. Para tanto, foi realizado levantamento bibliográfico e documental a fim de identificar referências que propuseram indicadores e/ou índices relacionados às águas pluviais urbanas, formulados para a realidade brasileira. Com base nas análises, verificou-se a importância dos dados que compõem determinado indicador serem de fácil obtenção e que o conjunto de indicadores/índice seja de fácil manuseio, sem deixar, porém, de atender ao objetivo. Os dados, a quantidade de indicadores e os pesos dos indicadores variam de acordo com o objetivo que se pretende alcançar com determinado conjunto de indicadores/índice. Para a realidade dos municípios brasileiros, indicadores de processo se apresentam como mais adequados, cabendo esforços para evolução dos mesmos dentro das oportunidades identificadas.
\end{abstract}

Palavras-chave: Drenagem Urbana; Ferramenta de Gerenciamento; SNIS-AP.

ABSTRACT: The effective management of urban stormwater in the Brazilian municipalities faces deadlocks, such as low availability and limited technical capacity in the responsible agencies and bureaus. An alternative to partially overcome these deadlocks would be an appropriate set of indicators. The purpose of this work is to evaluate the indicators related to urban stormwater formulated for the Brazilian reality and to identify existing barriers and opportunities for evolution. Therefore, a bibliographical survey was carried out in order to identify references that proposed the indicators and/or indexes related to urban stormwater, formulated for the Brazilian reality. Based on the analyses, the importance of the data that composes a given indicator being easily obtainable and that the set of indicators/indexes be easy to handle, without failing, however, to meet the objective, has been verified. The data, the amount of indicators and the value of these indicators vary according to the objective that is intended to be achieved with a given set of indicators/indexes. To the reality of Brazilian municipalities, process indicators are presented as more suitable, and efforts are needed for their evolution within the identified opportunities.

Keywords: Urban Drainage; Management Tool; SNIS-AP.

\section{INTRODUÇÃO}

0 acúmulo de águas pluviais urbanas foi, por muito tempo, visto como algo indesejável, de forma que as infraestruturas de drenagem eram implementadas para evacuar as águas pluviais para fora da área urbana o mais rápido possível (Bertrand-Krajewski, 2021; McGrane, 2016), sem necessidade de tratamento das mesmas (Bertrand-Krajewski, 2021). A partir da década de 1970, diante das visíveis desvantagens dessa abordagem, outras abordagens novas, mais abrangentes e integradas, foram sendo implementadas e aperfeiçoadas (Bertrand-Krajewski, 2021; Fletcher et al., 2015). Nesse

Recebido: Agosto 04, 2021. Revisado: Outubro 16, 2021. Aceito: Outubro 18, 2021. 
contexto, a necessidade da consideração de diversos aspectos e condições existentes no meio urbano torna o gerenciamento das águas pluviais complexo (Araújo et al., 2017).

A utilização de ferramentas que facilitem o processo de gerenciamento das águas pluviais urbanas e a tomada de decisão e, consequentemente, melhorem a drenagem e manejo das mesmas, tem-se ampliado nos últimos anos. No Brasil, de acordo com a Secretaria Nacional de Saneamento (SNS), um dos elementos essenciais para minimizar os impactos sofridos pelas populações em decorrência de eventos hidrológicos extremos é um adequado processo de planejamento e gestão dos serviços de drenagem das águas pluviais urbanas (Sistema Nacional de Informação sobre SaneamentoÁguas Pluviais, 2019).

Os indicadores e índices estão dentre as ferramentas utilizadas para planejamento e gerenciamento de processos e sistemas, como o sistema de águas pluviais (e.g. Bertilsson et al., 2019; Dizdaroglu, 2015; Huang et al., 2015; Igroufa et al., 2020; Miguez \& Veról, 2017; Romnée et al., 2015; Verma \& Raghubanshi, 2018). Indicadores medem fenômenos individuais e, quando combinados dois ou mais indicadores, estes formam um índice, o qual é baseado em pesos distintos dados aos indicadores constituintes (Verma \& Raghubanshi, 2018). Os indicadores, por terem como principais características a quantificação e a simplificação de informações sobre fenômenos complexos (van Bellen, 2005), têm sido utilizados para orientar os tomadores de decisão.

No entanto, apesar dos avanços no desenvolvimento de indicadores e índices relacionados às águas pluviais urbanas nos últimos anos, a inexistência de bancos de dados consolidados que permitam a utilização efetiva dessas ferramentas no gerenciamento, é ainda uma das grandes limitações. Klopp \& Petretta (2017) destacam como problemas práticos para sua aplicação de indicadores de desenvolvimento sustentável urbano a baixa disponibilidade de dados (padronizados, abertos e comparáveis), a falta de instituições fortes de coleta de dados em escala local e a capacidade técnica limitada nos órgãos e entidades responsáveis.

Estratégias para a gestão de águas pluviais urbanas precisam de informações e uma compreensão clara das possibilidades existentes, além de que uma decisão de alta qualidade demanda tempo e uma visão geral do problema (Barbosa et al., 2012). Morales-Torres et al. (2016) destacam que ferramentas para gestão de águas pluviais não devem considerar apenas construção e manutenção da infraestrutura, mas também considerar o tratamento de águas pluviais e custos de bombeamento, benefícios de coleta de água da chuva, serviços ecossistêmicos, melhorias de eficiência energética, benefícios de proteção contra enchentes, processos de qualidade da água e benefícios de isolamento de edifícios. De acordo com Vasconcelos \& Barbassa (2021) uma proposta efetiva de superação de barreiras e implantação efetiva da gestão sustentável de águas pluviais urbanas deve contemplar diversas estratégias, uma vez que estas são interdependentes, portanto, é ineficiente adotá-las isoladamente.

Os indicadores, ao apresentar dados e comparações, são úteis por inserir sustentabilidade à tomada de decisão e formulação de políticas (Streimikiene \& Šivickas, 2008). Nos primeiros anos do século XXI, os indicadores relacionados às águas pluviais urbanas tinham como principal objetivo a avaliação de desempenho de novas estruturas de drenagem de águas pluviais (e.g. Baptista et al., 2005; Kolsky \& Butler, 2002). Nesse mesmo período, métodos de análise multicritério, baseados em indicadores para auxiliar na resolução de problemas ambientais despontaram, sendo aplicados, também, na gestão de águas pluviais urbanas (e.g. Jia et al, 2013; Martin et al, 2007). Embora a evidente aplicação prática dos indicadores de desempenho, Kolsky \& Butler (2002) destacaram o desafio de encontrar indicadores que fossem válidos, relativamente fáceis de medir e úteis para o tomador de decisão - desafio, este, que perdura até os dias atuais, sendo os indicadores de processo uma alternativa. Dentre os indicadores de processo para o gerenciamento das águas pluviais no meio urbano, cabe destacar os indicadores e índices de previsão, os quais têm evoluído ao longo dos anos e auxiliam na gestão de risco de eventos extremos em áreas urbanas (e.g. Barroca et al., 2006; Bertilsson et al., 2019; Birgani \& Yazdandoost, 2014; Miguez \& Veról, 2017). No Brasil, são verificados estudos recentes relacionados a indicadores que avaliam a gestão das águas pluviais em um contexto mais amplo, envolvendo para além da drenagem e manejo de águas pluviais, aspectos institucionais, econômicos e sociais (e.g. Cavalcanti Filho, 2017; Silva, 2016).

Há, atualmente, uma elevada variedade de indicadores relacionados às águas pluviais para a realidade brasileira, desenvolvidos principalmente por pesquisadores. No entanto, os indicadores e índices desenvolvidos para fins de pesquisa não são, necessariamente, significativos para os formuladores de políticas e para a população em geral (Shields et al., 2002). Muitas vezes, os indicadores produzidos na academia são de difícil aplicação prática pelos gestores públicos (von Ancken et al., 2017). Além de impasses institucionais, no Brasil, a extensão territorial, que abriga 
elevada diversidade climática, fisiográfica, sociocultural e econômica, além de distintos processos de desenvolvimento urbanístico, dificulta que muitos indicadores ou índices sejam aplicados de forma uniforme, o que limita a formulação de políticas públicas efetivas pelo governo federal. Impasses semelhantes são relatados por Klopp \& Petretta (2017), que citam como um dos principais desafios para alcance dos objetivos do desenvolvimento sustentável urbano a aceitação de uma mesma meta de desenvolvimento sustentável para cidades com características distintas, com questões locais específicas.

No Brasil, a drenagem e manejo de águas pluviais é o componente do saneamento básico menos assistido em termos de regulação e regulamentação direta pelos Titulares, que são os municípios (Colombelli, 2018). Na maior parte dos municípios brasileiros $(98,9 \%)$ os prestadores do serviço de manejo e drenagem de águas pluviais pertencem às próprias prefeituras municipais, as quais são as responsáveis pelo serviço (Sistema Nacional de Informação sobre Saneamento-Águas Pluviais, 2019) e, em maioria, carecem de uma estruturação institucional adequada (Colombelli, 2018) e técnicos capacitados para conceber, dimensionar e gerir os sistemas de drenagem de águas pluviais com foco nas bacias hidrográficas, além da questão dos conceitos de sustentabilidade na drenagem urbana (Oneda, 2018). Nesse sentido, os indicadores são ferramentas que, se adequadas à realidade municipal e compreensíveis aos gestores e agentes públicos, apresentam potencial para serem utilizadas no gerenciamento das águas pluviais urbanas.

Atualmente, a utilização de indicadores na gestão de águas pluviais não é comumente observada nos governos municipais. A falta de uma lista abrangente de indicadores para o desenvolvimento urbano sustentável dificulta a avaliação da sustentabilidade urbana em contextos variados (Kaur \& Garg, 2019). O Brasil ainda se encontra em uma etapa de consolidação de indicadores básicos de determinados setores que fazem parte do ambiente urbano. Assim, o objetivo deste trabalho é avaliar os indicadores relacionados às águas pluviais urbanas formulados para a realidade brasileira e identificar os entraves existentes e as oportunidades de avanço. Espera-se que esse trabalho contribua para a evolução dos sistemas de indicadores para gerenciamento das águas pluviais urbanas no Brasil, além de destacar quais tipos de indicadores podem ser mais explorados e formas de organizá-los, a fim de auxiliar, efetivamente, os gestores na tomada de decisão.

\section{METODOLOGIA}

Para alcançar o objetivo proposto, esse estudo foi realizado com base em levantamento bibliográfico e documental, a partir do qual foi realizada discussão em duas etapas principais: 1) Análise dos indicadores/índices propostos nas bibliografias; e 2) Identificação dos principais entraves e de oportunidades para que os indicadores relativos às águas pluviais urbanas sirvam efetivamente como suporte aos tomadores de decisão.

Com vistas a identificar os indicadores e índices relacionados às águas pluviais urbanas existentes formulados para a realidade brasileira, foi realizado um levantamento bibliográfico e documental. 0 levantamento bibliográfico foi realizado nas bases de dados Google Scholar, Web of Science (WoS) e Scopus, no segundo semestre de 2020 e primeiro semestre de 2021. As bibliografias pesquisadas selecionados foram restritas a artigos revisados por pares, dissertações e teses que formularam e/ou propuseram indicadores e/ou índices relacionados às águas pluviais em ambiente urbano. As seguintes palavras-chave foram pesquisadas: "indicador", "índice”, "águas pluviais", "inundação", "drenagem urbana" e "Brasil". Considerou-se variações na grafia das palavras (por exemplo, água da chuva) e também combinações entre os termos, com o objetivo de refinar os resultados. Foram consideradas bibliografias em inglês e em português. 0 período para o qual foram encontradas referências de indicadores e índices relacionados às águas pluviais urbanas existentes formulados para a realidade brasileira para a realidade brasileira foi compreendido entre os anos 2004 e 2020. 0 levantamento documental consistiu na identificação de Planos Municipais, de manejo de águas pluviais ou de saneamento que contemplassem o componente águas pluviais e que possuíssem indicadores ou índices. Foram pesquisados Planos Municipais de capitais de estados brasileiros das cinco regiões políticas, visando contemplar elementos de diferentes realidades brasileiras.

Para a identificação dos entraves existentes e de oportunidades de evolução, inicialmente foram analisados, nos indicadores e índices propostos nas bibliografias e Planos Municipais, os seguintes aspectos: 1) Dados de entrada: número de indicadores que compõem o conjunto de indicadores ou índice, quantidade de dados requerida, tipo do indicador (quantitativo ou qualitativo), grau de dificuldade de obtenção dos dados e complexidade de manipulação do indicador/índice. Para atribuição do grau de dificuldade e complexidade foram considerados: Fácil - envolvem cálculos 
matemáticos simples similares ao do SNIS; Intermediário - envolvem cálculos matemáticos menos complexos; Difícil - envolvem cálculos matemáticos complexos, manuseio de softwares e/ou análises laboratoriais.; 2) Nível de agregação (indicador simples - mede o fenômeno individualmente; índice um único resultado derivado da agregação de dois ou mais indicadores simples) e de ponderação; 3) Variáveis de mensuração (indicadores de desempenho: medem a performance de determinado fenômeno ou condição ao longo de um espaço de tempo; indicadores de processo: descrevem o estado do sistema de drenagem); e 4) Atendimento à abrangência estipulada pela Lei Federal n 11.445/2007 (atualizada pela Lei Federal $\left.n^{\circ} 14.026 / 2020\right)$ para sistema de indicadores: áreas temáticas: 1) Sanitária; 2) Epidemiológica; 3) Ambiental; e 4) Socioeconômica (Brasil, 2007, 2020).

É importante ressaltar que o objetivo desta revisão foi identificar os indicadores e índices relacionados às águas pluviais urbanas existentes formulados para a realidade brasileira, que não são, necessariamente, os indicadores e índices mais avançadas no tema.

Para maior clareza, as seguintes definições de termos se aplicam a este artigo: 1) águas pluviais urbanas são as águas geradas por precipitações em ambientes urbanizados, resultantes do escoamento em superfícies impermeáveis e/ou saturadas; 2) drenagem e manejo das águas pluviais urbanas são "constituídos pelas atividades, pela infraestrutura e pelas instalações operacionais de drenagem de águas pluviais, transporte, detenção ou retenção para o amortecimento de vazões de cheias, tratamento e disposição final das águas pluviais drenadas, contempladas a limpeza e a fiscalização preventiva das redes" (Brasil, 2020); 3) gerenciamento de águas pluviais urbanas se refere à aplicação de conhecimentos, técnicas e metodologias que visam orientar as decisões do gestor público no que se relaciona às águas pluviais em ambientes urbanizados; 4) gestão de águas pluviais urbanas se refere ao planejamento e gerenciamento das águas pluviais urbanas, criação de políticas e legislação, baseadas em princípios, estratégias e diretrizes de ações e procedimentos para garantir que as águas pluviais em áreas urbanas causem menos prejuízos à população e ao meio ambiente, agregando uma melhor qualidade de vida e reduzindo os gastos do poder público no pós-evento.

\section{RESULTADOS E DISCUSSÃO}

\section{Indicadores e índices existentes e/ou aplicáveis à realidade brasileira}

Foram identificadas vinte e sete (27) bibliografias que propuseram conjuntos de indicadores ou índices relacionados às águas pluviais urbanas formulados para a realidade brasileira. Destaca-se que a maioria foi publicada na última década (2010 a 2020). Na Tabela 1 são apresentadas as bibliografias identificadas o número de indicadores e a quantidade de dados requeridos para obtenção dos resultados dos indicadores/índices, além da apresentação desses mesmos dados dos Planos Municipais analisados.

Tabela 1 - Informações dos indicadores e índices analisados.

\begin{tabular}{|c|c|c|c|c|}
\hline Id & Nome & $\begin{array}{l}\text { Número de } \\
\text { indicadores }\end{array}$ & $\begin{array}{l}\text { Quantidade } \\
\text { de dados } \\
\text { requerida }\end{array}$ & Autor e Ano \\
\hline \multicolumn{5}{|c|}{ Bibliografias } \\
\hline 1 & $\begin{array}{l}\text { Indicadores para a avaliação de sistemas de } \\
\text { drenagem urbana }\end{array}$ & 13 & 20 & Castro et al. (2004) \\
\hline 2 & $\begin{array}{c}\text { Indicadores para avaliação de sistemas de } \\
\text { drenagem }\end{array}$ & 10 & 28 & Silva de Brito (2006) \\
\hline 3 & $\begin{array}{l}\text { Indicadores da Drenagem Urbana Sustentável } \\
\text { (IDUS) }\end{array}$ & 29 & $* * *$ & Marques (2006) \\
\hline 4 & Índice de Vulnerabilidade Composto (CVI) & 15 & 29 & Szlafsztein \& Sterr (2007) \\
\hline 5 & Índice de Risco de Cheia (IRC) & 7 & 10 & Zonensein (2007) \\
\hline 6 & $\begin{array}{l}\text { Índices de desempenho e de custos de } \\
\text { sistemas de drenagem urbana }\end{array}$ & 14 & 16 & Moura et al. (2009) \\
\hline 7 & $\begin{array}{c}\text { Indicadores para a avaliação dos efeitos da } \\
\text { urbanização nos corpos de água }\end{array}$ & 8 & 24 & Castro et al. (2009) \\
\hline 8 & Índice de Risco de Alagamento (IRA) & 4 & 6 & Holz (2010) \\
\hline 9 & $\begin{array}{l}\text { Indicadores de desempenho para sistemas de } \\
\text { infiltração de águas pluviais urbanas }\end{array}$ & 20 & 27 & Moura et al. (2010) \\
\hline
\end{tabular}


Tabela 1 - Continuação...

\begin{tabular}{|c|c|c|c|c|}
\hline Id & Nome & $\begin{array}{l}\text { Número de } \\
\text { indicadores }\end{array}$ & $\begin{array}{l}\text { Quantidade } \\
\text { de dados } \\
\text { requerida }\end{array}$ & Autor e Ano \\
\hline 10 & $\begin{array}{l}\text { Índice de vulnerabilidade urbana a eventos } \\
\text { extremos de chuva }\end{array}$ & 1 & 2 & Perez (2013) \\
\hline 11 & $\begin{array}{l}\text { Indicadores de sustentabilidade para } \\
\text { avaliação do sistema de drenagem urbana }\end{array}$ & 15 & 15 & Ripol e Silva et al. (2013) \\
\hline 12 & Índice de Fragilidade do Sistema & 26 & 26 & Santos Júnior (2014) \\
\hline 13 & $\begin{array}{l}\text { Índices de resiliência hídrica e de perigo para } \\
\text { gestão do risco de inundações urbanas }\end{array}$ & 2 & 8 & Rotava (2014) \\
\hline 14 & $\begin{array}{l}\text { Indicadores de sustentabilidade para manejo } \\
\text { de águas pluviais }\end{array}$ & 54 & 96 & Silva (2016) \\
\hline 15 & $\begin{array}{l}\text { Índice de vulnerabilidade a inundações de } \\
\text { municípios urbanos }\end{array}$ & 26 & 26 & Rasch (2016) \\
\hline 16 & $\begin{array}{l}\text { Índice de vulnerabilidade a inundações } \\
\text { repentinas e Índice de vulnerabilidade a } \\
\text { escorregamentos }\end{array}$ & 18 & 18 & Debortoli et al. (2017) \\
\hline 17 & Índice de Resiliência de Inundações (FRI) & 5 & 7 & Miguez \& Veról (2017) \\
\hline 18 & $\begin{array}{l}\text { Indicadores do sistema de drenagem urbana } \\
\text { e manejo de águas pluviais }\end{array}$ & 44 & 53 & Cavalcanti Filho (2017) \\
\hline 19 & $\begin{array}{l}\text { Índice de resiliência a inundações urbanas } \\
\text { (UFRI) }\end{array}$ & 3 & 9 & Rezende et al. (2019) \\
\hline 20 & $\begin{array}{l}\text { Índice espacializado de resiliência a } \\
\text { inundações urbanas (S-FRESI) }\end{array}$ & 5 & 5 & Bertilsson et al. (2019) \\
\hline 21 & Índice de Restauração de Rio Urbano (URRIX) & 4 & 15 & Veról et al. (2019) \\
\hline 22 & $\begin{array}{l}\text { Indicadores da avaliação da conservação } \\
\text { ambiental de riachos urbanos }\end{array}$ & 9 & 12 & Cometti et al. (2019) \\
\hline 23 & Índice Simplificado de Risco de Cheias (SFRI) & 2 & 7 & Miranda et al. (2019) \\
\hline 24 & $\begin{array}{l}\text { Indicadores de desempenho de sistemas de } \\
\text { drenagem urbana }\end{array}$ & 16 & 19 & Mendonça \& Souza (2019) \\
\hline 25 & $\begin{array}{c}\text { Índice de Risco de Inundação para a } \\
\text { Capacidade de Recuperação Socioeconômica } \\
\text { (Ri-SoRCI) }\end{array}$ & 2 & 11 & Rezende et al. (2020) \\
\hline 26 & Índice de Risco de Inundação (IRI) & 2 & 9 & Pereira et al. (2020) \\
\hline 27 & índice de qualidade de vida melhorado (iQoL) & 1 & 11 & McClymont et al. (2020) \\
\hline \multicolumn{5}{|c|}{ Planos Municipais } \\
\hline 1 & Indicadores de metas de drenagem & 8 & 11 & $\begin{array}{l}\text { Plano Municipal de Saneamento } \\
\text { Básico de Belém (Belém, 2020) }\end{array}$ \\
\hline 2 & $\begin{array}{l}\text { Indicadores de desempenho do sistema de } \\
\text { águas pluviais }\end{array}$ & 18 & 30 & $\begin{array}{c}\text { Plano Municipal de Saneamento } \\
\text { Básico de Porto Alegre (Porto } \\
\text { Alegre, 2015) } \\
\end{array}$ \\
\hline 3 & $\begin{array}{l}\text { Indicadores para o serviço de drenagem e } \\
\text { manejo de águas pluviais urbanas }\end{array}$ & 4 & 7 & $\begin{array}{l}\text { Plano Municipal de Saneamento } \\
\text { Básico de Natal (Natal, 2015) }\end{array}$ \\
\hline 4 & Índice de Drenagem Urbana & 1 & 2 & $\begin{array}{c}\text { Plano Municipal de Saneamento } \\
\text { Básico de Belo Horizonte (Belo } \\
\text { Horizonte, 2020) } \\
\end{array}$ \\
\hline 5 & $\begin{array}{l}\text { Indicadores utilizados para o sistema de } \\
\text { drenagem urbana }\end{array}$ & 19 & 31 & $\begin{array}{l}\text { Plano Distrital de Saneamento } \\
\text { Básico do Distrito Federal } \\
\text { (Distrito Federal, 2017) }\end{array}$ \\
\hline 6 & $\begin{array}{l}\text { Indicadores de desempenho do sistema de } \\
\text { drenagem urbana e manejo das águas } \\
\text { pluviais }\end{array}$ & 31 & 35 & $\begin{array}{l}\text { Plano de Águas Pluviais de São } \\
\text { Paulo (São Paulo, 2012) }\end{array}$ \\
\hline
\end{tabular}

\section{Dados de entrada}

A quantidade de indicadores necessária para a composição dos conjuntos de indicadores e índices avaliados é diversa, variando de 1 a 54 indicadores. Essa diversidade pode ser observada na Figura 1A, onde também se observa que a maior parte das bibliografias e Planos Municipais requerem de 1 a 10 indicadores. No que tange à quantidade de dados requeridos para o cálculo dos conjuntos de indicadores simples e índices avaliados (Figura 1B), é possível observar que a maior quantidade das 
bibliografias e Planos Municipais requerem de 1 a 15 dados para o cálculo dos conjuntos de indicadores e índices avaliados. Esse comportamento, em que as quantidades de indicadores e de dados requeridos são similares, é esperado, uma vez que os indicadores relacionados às águas pluviais urbanas geralmente não requerem muitos dados para seu cálculo.

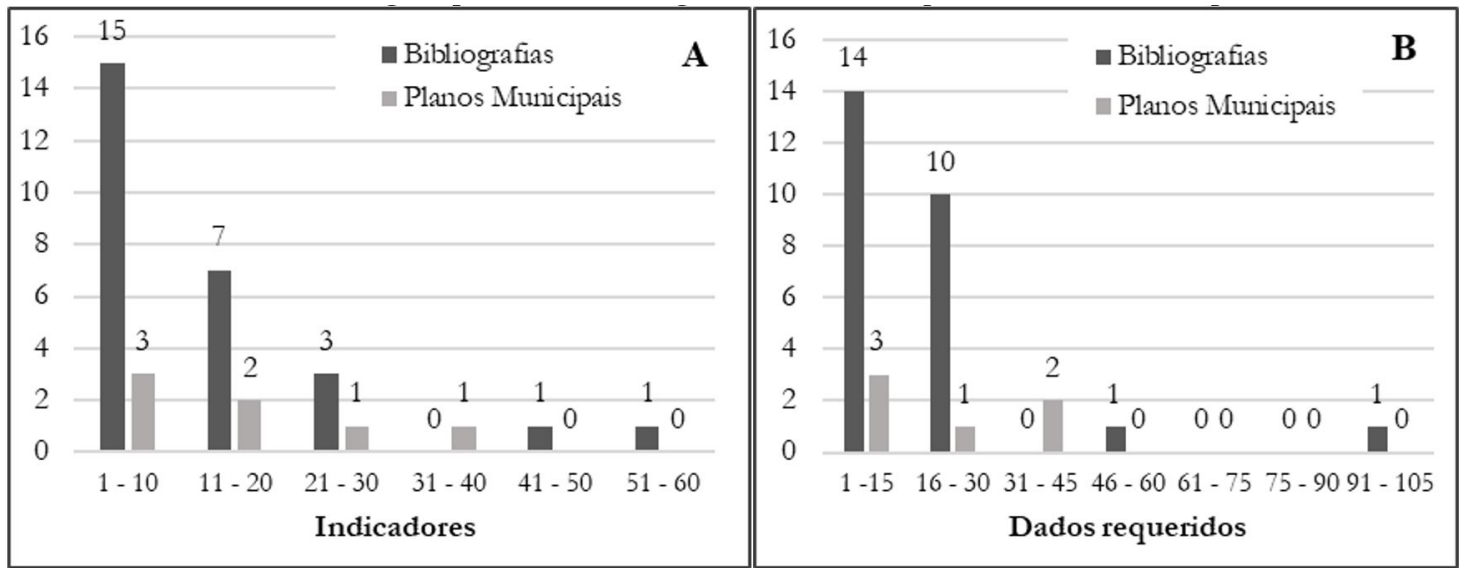

Figura 1 - Distribuição da quantidade de indicadores que compõem os conjuntos de indicadores ou índices $(\mathrm{A})$; Quantidade de dados requeridos para cálculo dos conjuntos de indicares ou índices (B).

Em relação aos dados de entrada dos conjuntos de indicadores e índices avaliados, observou-se a predominância da requisição de dados qualitativos, tanto para as bibliografias quanto para os Planos Municipais. Dos seis Planos Municipais avaliados, apenas um utiliza dados qualitativos e quantitativos para a quantificação dos indicadores, enquanto os demais utilizam apenas dados quantitativos. Na Figura 2 é apresentada a quantidade de dados quantitativos e qualitativos necessários para o cálculo dos indicadores simples ou índice de cada bibliografia.

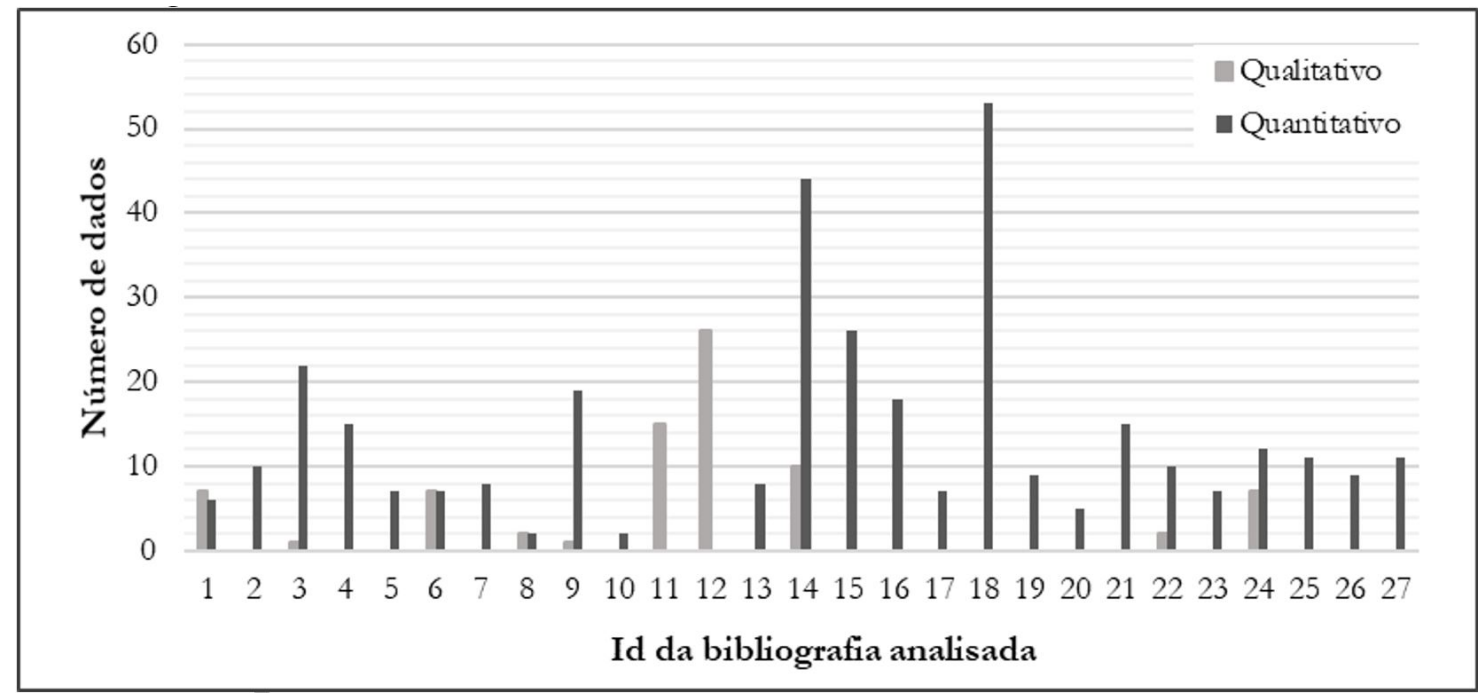

Figura 2 - Quantificação dos indicadores quantitativos e qualitativos por bibliografia.

Ainda, no que tange aos dados de entrada dos indicadores/índices das bibliografias, os mesmos são, em maioria, de difícil obtenção, enquanto os dados de entrada dos indicadores/índices dos Planos Municipais são de fácil obtenção (Figura 3A). A dificuldade relacionada a obtenção dos dados de entrada dos indicadores/índices das bibliografias está relacionado, em grande maioria, ao fato da utilização de softwares para obtenção de alguns dados ou informações. No entanto, durante a formulação dos indicadores/índices, devem ser consideradas as capacidades computacionais e técnicas de quem irá, na prática, manusear os indicadores/índices. No caso dos Planos Municipais, em que é sabido que os indicadores/índices serão utilizados pela gestão pública e quem irá manusear os 
mesmos serão técnicos, os dados necessários para o cálculo são de fácil obtenção, logo, não inviabilizam o uso dos mesmos.

Diferentemente do observado em relação à obtenção dos dados de entrada dos indicadores/índices das bibliografias, a manipulação dos indicadores/índices, tanto das bibliografias quanto dos Planos Municipais apresentou, em maioria, um grau de complexidade de manipulação fácil (Figura 3B). Esse resultado pode ser atribuído as fórmulas de cálculo dos indicadores/índices, que costumam ser simples, logo, com os dados de entrada conhecidos, o cálculo do indicador não apresenta complexidade elevada.
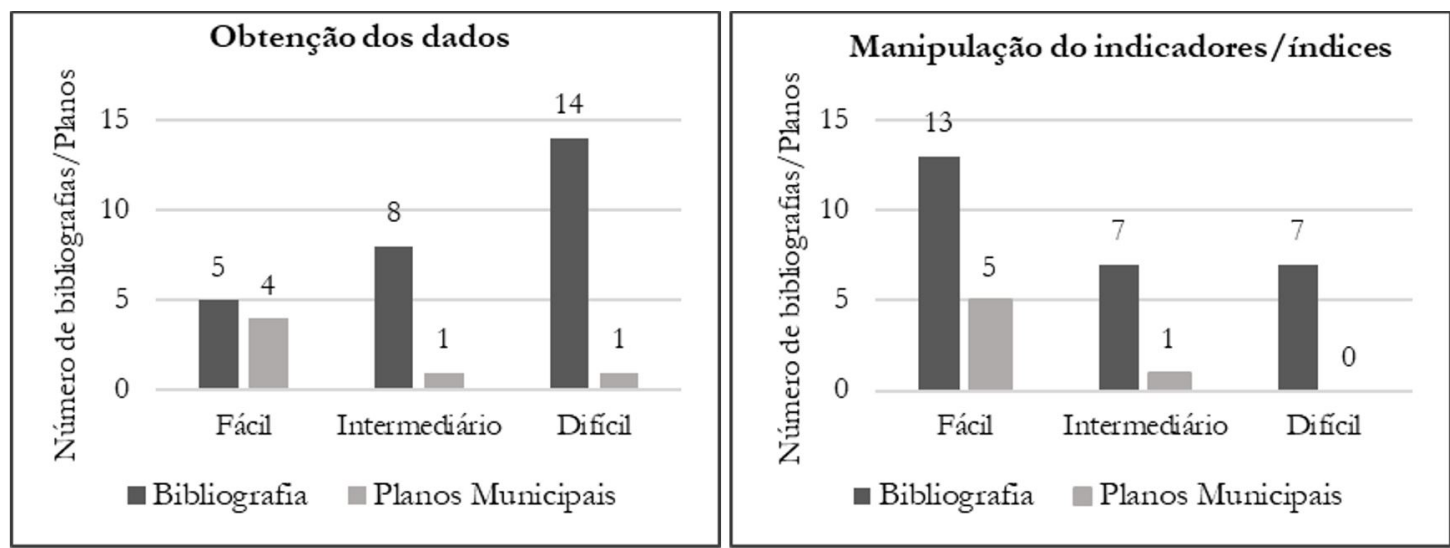

Figura 3 - Grau de dificuldade de obtenção dos dados (A); Complexidade de manipulação dos indicadores/índices (B).

A quantidade de dados e de indicadores e os pesos dependem do objetivo que se quer alcançar com determinado conjunto de indicadores/índice. No entanto, para fins de gerenciamento municipal das águas pluviais em ambiente urbano, é fundamental que os dados que compõe sejam de fácil obtenção e que o conjunto de indicadores/índice sejam de fácil manuseio, além de atender ao objetivo para o qual foi desenvolvido.

\section{Agregação e ponderação}

Das vinte e sete (27) bibliografias avaliadas, dez (10) propuseram conjuntos de indicadores e dezessete (17) propuseram índices relacionados às águas pluviais urbanas formulados para a realidade brasileira. Dentre os seis (6) Planos Municipais analisados, apenas um propôs índice. As publicações relacionadas à previsão/vulnerabilidade a eventos hidrológicos foram agregadas em nível de índice, enquanto os indicadores das demais publicações, majoritariamente, foram organizados e apresentados em nível de indicadores. Conforme Hammond et al. (1995), indicadores altamente agregados, ao comprimir e simplificar as informações, comunicam-se com mais eficácia. Contudo, criar um indicador único ou índice na área socioambiental resulta em uma leitura simplista, considerando a complexidade que envolve essa área (Sepe \& Gomes, 2008).

A previsão e vulnerabilidade a eventos hidrológicos são apenas uma parte de todo o conteúdo que abrange o gerenciamento de águas pluviais urbanas. Sendo estes indicadores de previsão/vulnerabilidade mais específicos, menos subjetividade está intrínseca nos índices formados a partir destes, quando comparados a índices que devem apresentar como resultados um panorama do gerenciamento das águas pluviais urbanas como um todo. No entanto, Rasch (2016) atenta para os perigos de avaliar a vulnerabilidade usando índices socioeconômicos em países de renda com altos níveis de desigualdade, onde a presença de populações vulneráveis em assentamentos informais em municípios altamente desenvolvidos pode ser mascarada.

No que tange a ponderação, muitos conjuntos de indicadores simples das bibliografias analisadas não apresentam ponderação (80\%), enquanto para os índices esse percentual foi de 47\%. Três (3) índices avaliados atribuíram ponderação igualitária. Rezende et al. (2020), que assumiu ponderação igualitária em seu estudo, destaca que definir a importância relativa de cada termo é uma etapa importante do processo de desenvolvimento de um índice, mas deve ser feito após a validação do método proposto. Além disso, o processo de definição dessa importância relativa é algo que pode variar de caso para caso e que deve ser prerrogativa dos tomadores de decisão (Rezende et al., 2020). 
Dentre os indicadores/índices que foram submetidos a ponderação os indicadores simples com maior peso foram: impacto sobre as vazões de jusante (Castro et al., 2004), população afetada (Szlafsztein \& Sterr, 2007), atendimento aos objetivos de drenagem (Moura et al., 2009), custos de construção e custos de operação e manutenção (Moura et al., 2010), profundidade máxima da inundação (Miguez \& Veról, 2017), atendimento a legislação (Mendonça \& Souza, 2019) e declividade de densidade demográfica (Pereira et al., 2020). Dentre os indicadores simples com menor peso, destacam-se: possibilidade da utilização para o desempenho de outras funções técnicas na estrutura de drenagem (Castro et al., 2004; Moura et al., 2009), orçamento municipal e pobreza (Szlafsztein \& Sterr, 2007), uso do solo (Moura et al., 2010), saneamento inadequado (Miguez \& Veról, 2017), riscos e vulnerabilidade à saúde pública (Mendonça \& Souza, 2019) e educação, vegetação e pedologia (Pereira et al., 2020). A ampla diversidade existente entre os indicadores de maior peso e entre os indicadores de menos peso pode ser explicada pelo fato de que os pesos vão variar de acordo com o objetivo do indicador/índice; o que pode ser de maior ponderação em um indicador, não necessariamente será em outro. De acordo com Miguez \& Veról (2017), a determinação dos pesos é geralmente complexa e muito frequentemente influenciada por percepções pessoais e, apesar das dificuldades de se estabelecer uma metodologia livre de subjetividade, é necessário estabelecer uma base teórica ou algum tipo de raciocínio lógico para garantir a credibilidade e aceitação na atribuição de pesos.

Em relação aos indicadores/índice do Planos Municipais, nenhum foi submetido ao processo de ponderação. Cabe destacar que o Índice de Drenagem Urbana do Plano Municipal de Saneamento Básico de Belo Horizonte compõem o Índice de Salubridade Ambiental (ISA), o qual possui ponderação (Belo Horizonte, 2020). O Indicador de Salubridade Ambiental (ISA) foi criado, em 1999, pelo Conselho Estadual de Saneamento (Conesan) do Estado de São Paulo, com o objetivo de medir o nível de salubridade ambiental dos municípios paulistas, sendo difundido pelo Brasil com alterações em sua formulação original, de acordo com as características do local a ser avaliado, como é sugerido pelo manual que o originou (Teixeira et al., 2018). No caso da ponderação dos índices formadores do ISA do Plano Municipal de Saneamento Básico de Belo Horizonte, o Índice de Drenagem Urbana foi o que recebeu o maior peso, enquanto o Índice de Águas de Abastecimento recebeu o menor peso. devido a situação atual de quase universalização desse serviço (Belo Horizonte, 2020). No entanto, a ponderação pelo nível de universalização do serviço não é unanimidade entre Planos de Saneamento. No ISA do Plano Estadual de Saneamento Básico do Rio Grande do Sul, por exemplo, os indicadores com maior peso foram os de abastecimento de água, esgotamento sanitário e resíduos sólidos, enquanto os indicadores com menor peso foram os indicadores institucional e socioeconômico, sendo que o indicador de drenagem urbana apresentou peso intermediário (Rio Grande do Sul, 2021).

Em relação ao nível de agregação e ponderação, de acordo com Bauler (2012), um aspecto a se considerar e que desempenha um papel importante em influenciar as decisões, são as percepções dos atores da política sobre os resultados e o julgamento subjetivo sobre os indicadores. Se o nível de compreensão do resultado de determinado conjunto de indicadores for reduzido, as decisões políticas podem aumentar as disparidades econômicas, os danos ambientais e diminuir as possibilidades de sustentabilidade a longo prazo (Mayer, 2008).

\section{Variáveis de mensuração}

Os indicadores/índices foram classificados como de desempenho ou como de processo. Nas bibliografias é predominante os indicadores de processo (vinte (20) publicações), enquanto nos Plano Municipais é predominante os indicadores de desempenho (quatro (4) planos).

De acordo com Kolsky \& Butler (2002) indicadores de processo podem ser muito mais práticos do que os indicadores de desempenho em si, tanto em medição, pois costumam ser difíceis de medir, quanto em uso, pois eles podem descrever o problema, mas não o que fazer a respeito. Os indicadores de processo mais comuns nas publicações apresentadas foram os indicadores de previsão, que são desenvolvidos em face aos tomadores de decisão locais requererem ferramentas para uma melhor avaliação da vulnerabilidade das cidades às inundações, para além de abordagens apenas qualitativas (Barroca et al., 2006).

No Brasil, o conhecimento e entendimento acerca do sistema de águas pluviais, funcionamento e gestão do mesmo é insipiente, fato esse apontado pelo Sistema Nacional de Informação sobre Saneamento-Águas Pluviais (2019), em que aproximadamente um terço dos municípios (35,3\%) não forneceram os dados para o cálculo dos indicadores do SNIS-AP e, dentre os que forneceram, é possível identificar dificuldade de compreensão e preenchimento por parte de vários municípios. Nesse 
sentido, indicadores de processo, com um menor nível de agregação, podem ser mais interessantes para o momento atual, de forma a fomentar a compreensão dos problemas principais existentes. Os indicadores de desempenho apresentam maior aplicação para verificar um sistema ou condição já existente e de comportamento conhecido ou para avaliar qual sistema é mais adequado a uma condição conhecida.

\section{Atendimento à abrangência estipulada pela legislação federal}

A Lei Federal $n^{\circ} 11.445 / 2007$, atualizada pela Lei Federal $n^{\circ} 14.026 / 2020$, estabelece que os serviços públicos de saneamento básico, incluindo o manejo e drenagem de águas pluviais, devem observar o Plano Municipal de Saneamento Básico ou o Plano Diretor de Drenagem Urbana. Estes documentos devem abranger, dentre os conteúdos mínimos, um diagnóstico da situação e de seus impactos nas condições de vida, utilizando sistema de indicadores que contemple as áreas temáticas: 1) Sanitária; 2) Epidemiológica; 3) Ambiental; e 4) Socioeconômica, apontando as causas das deficiências identificadas (Brasil, 2007, 2020).

Dentre as publicações avaliadas apresentadas na Tabela 1, Marques (2006), Ripol e Silva et al. (2013), Santos Júnior (2014), Silva (2016) e Cavalcanti Filho (2017) propuseram sistemas de indicadores que representassem o sistema de drenagem e que pudessem ser utilizados pela administração pública no gerenciamento de águas pluviais urbanas. Ao analisar se esses sistemas de indicadores contemplam as áreas temáticas (Sanitária, Epidemiológica, Ambiental e Socioeconômica) estipuladas na Lei Federal n 11.445/2007, atualizada pela Lei Federal n 14.026/2020 (Brasil, 2007, 2020), verifica-se que os indicadores propostos por Marques (2006) contemplam as quatro áreas temáticas requeridas. No entanto, há lacunas na gestão de risco, como mapeamento das áreas impactadas por esse tipo de desastre, população atingida e existência de plano de emergência. Os indicadores simples propostos por Ripol e Silva et al. (2013), Santos Júnior (2014) e Silva (2016) não contemplam em sua totalidade as áreas temáticas requeridas pela legislação, enquanto os indicadores simples propostos por Cavalcanti Filho (2017) atendem ao básico requerido pela legislação. No entanto, não basta que os conjuntos de indicadores atendam a determinados requisitos, eles também devem ser aplicáveis. von Ancken et al. (2017), ao aplicarem os indicadores elencados por Silva (2016), observaram que muitos dos indicadores propostos são de difícil determinação, uma vez que a aquisição dos dados necessários aos cálculos desses indicadores exigem o uso de equipamentos específicos, softwares ou ações de monitoramento.

Assim como observado nos indicadores/índices das bibliografias analisadas, os indicadores/índices dos Planos Municipais não contemplam as áreas temáticas estipuladas na Lei Federal $n^{\circ} 11.445 / 2007$, atualizada pela Lei Federal $n^{\circ}$ 14.026/2020 (Brasil, 2007, 2020), com exceção dos indicadores de desempenho do sistema de drenagem urbana e manejo das águas pluviais propostos no Plano de Águas Pluviais de São Paulo (São Paulo, 2012).

Cabe aqui destacar que, apesar da Lei Federal $n^{\circ} 11.445 / 2007$, atualizada pela Lei Federal $n^{\circ} 14.026 / 2020$, estabelecer áreas temáticas mínimas que um conjunto de indicadores deve abranger, o próprio conjunto de indicadores do SNIS-AP não abrange as quatro áreas temáticas. A divulgação dos dados sobre manejo e drenagem de águas pluviais é realizada por meio de indicadores (25) e informações (125) em quatro campos de análise: 1) Econômico-financeiras e administrativas; 2) Infraestrutura; 3) Manutenção; e 4) Gestão de risco (Sistema Nacional de Informação sobre Saneamento-Águas Pluviais, 2019). Apesar dos nomes distintos das áreas temáticas propostas pela Lei Federal n 11.445/2007, atualizada pela Lei Federal n 14.026/2020 (Brasil, 2007, 2020), várias delas se complementam, com exceção da área temática Epidemiológica, que não possui nenhum indicador diretamente relacionado no conjunto de indicadores do SNIS-AP.

Apesar da grande maioria das bibliografias e Planos Municipais analisados não contemplarem as quatro áreas temáticas mínimas que um conjunto de indicadores deve abranger, conforme a Lei Federal $n^{\circ}$ 11.445/2007, atualizada pela Lei Federal n 14.026/2020 (Brasil, 2007, 2020), essa não é uma exigência inatingível. Foi possível verificar que alguns conjuntos de indicadores/índices contemplavam algumas das áreas temáticas, enquanto outros contemplavam outras áreas temáticas, de forma que para todas as áreas temáticas há indicadores com dados de fácil obtenção e que são de fácil manuseio. 
Entraves e oportunidades de evolução nos conjuntos de indicadores

A ausência de conhecimento sobre a realidade das águas pluviais urbanas no Brasil e sobre as alternativas para gerir estas águas pode ser provida com um conjunto de indicadores que seja consolidado. 0 conhecimento formulado e aprimorado por pesquisadores impulsiona a formulação de políticas públicas, mecanismos e ferramentas que auxiliam gestores públicos.

Em 2015, o governo brasileiro iniciou a coleta sistematizada de dados sobre manejo e drenagem de águas pluviais, a nível municipal, realizada por meio do SNIS-AP e, mesmo que tardia e com qualidade relativa de informações, os resultados permitem uma visualização geral da situação nacional em relação a esse setor, que até o momento não era possível. 0 conjunto de indicadores do SNIS-AP pode ser considerado um conjunto de indicadores mínimos essenciais. No entanto, há problemas relacionados à confiabilidade dos dados informados no sistema, que está atrelada ao fornecimento destes (uma vez que o preenchimento é de responsabilidade das autoridades municipais, nem sempre tecnicamente aparelhadas para tanto, além da recorrente ruptura na constituição das equipes locais) e à forma diversificada como os conceitos e termos técnicos de drenagem são apropriados em cada região do país (Sistema Nacional de Informação sobre Saneamento-Águas Pluviais, 2019). Apesar dos entraves relacionados aos dados declarados e a quem os declara, que limitam o uso dessas informações de forma ampla, os diagnósticos de drenagem e manejo das águas pluviais do SNIS, no longo prazo, constituirão séries históricas de diversas informações que darão nova dimensão à tomada de decisão nesse setor.

Para além dos indicadores do SNIS, indicadores específicos para cada realidade municipal são importantes, por agregarem características específicas e auxiliarem na tomada de decisão municipal de acordo com os objetivos específicos de cada município para com o serviço de águas pluviais urbanas. A seguir são apresentadas as principais oportunidades identificadas em relação a aspectos técnicos e aspectos legais envolvendo indicadores das águas pluviais urbana, além de experiências que podem ser transpostas para a realidade brasileira.

\section{Aspectos técnicos}

0 desenvolvimento dos indicadores/índices envolvem diversas etapas. Em relação ao formato de apresentação dos indicadores (nível de agregação e variáveis de mensuração), diante da limitação existente no que tange a profissionais capacitados na área de águas pluviais nos municípios, o mais adequado é que os indicadores simples sejam menos agregados, permitindo ao analista dos resultados finais mais informações para a tomada de decisão; também, como atualmente não há bases de dados consolidadas em nenhuma região do país sobre águas pluviais urbanas e por muitos locais não terem conhecimento efetivo sobre os principais problemas envolvendo águas pluviais urbanas, o uso de indicadores de desempenho se torna menos recomendável.

Outro ponto sensível identificado é que muitos conjuntos de indicadores exigem informações complexas, que demandam ferramentas muito específicas para que sejam obtidas as informações necessárias. Quando a finalidade é a constituição de uma ferramenta para utilização pelo poder público, é fundamental que ela seja de fácil manuseio e exija informações de obtenção relativamente fácil, dada a capacidade limitada do quadro de servidores públicos para a área de águas pluviais existente nos municípios.

A normalização, que é uma etapa realizada antes da ponderação, é interessante que seja realizada, independentemente se há ou não agregação dos indicadores simples, isso porque atribui comensurabilidade aos indicadores, permitindo assim compreender qual aspecto está mais crítico, ou que necessita de ações imediatas, por exemplo. A comensurabilidade permite a comparação entre resultados entre indicadores de forma coerente, auxiliando os tomadores de decisão.

Quanto a ponderação dos indicadores, é importante observar que essa etapa é mais comumente utilizada e necessária quando da agregação de indicadores em índices, tornando transparente o cálculo. Há diversos métodos de ponderação existentes, no entanto, em muitos documentos e bibliografias não é detalhado o método utilizado. É importante, principalmente em conjuntos de indicadores para fins de usos públicos, que seja discriminado como foi realizada essa etapa, do contrário, o nível de compreensão do resultado de determinado conjunto dos indicadores/índices pelos tomadores de decisão pode ser reduzido, podendo afetar negativamente nas decisões tomadas.

Em relação aos Planos Municipais, a manutenção e o monitoramento de indicadores permitem, no longo prazo, a constituição de uma série histórica de seus resultados, importante objeto para tomadores de decisão, como no caso do gerenciamento de drenagem e manejo de águas pluviais. Diferentemente do SNIS, que reconhece que a coleta de dados e informações deu início à uma possível 
série histórica (Sistema Nacional de Informação sobre Saneamento-Águas Pluviais, 2019), alguns decisores, como o governo de São Paulo, por exemplo, consideram que o número de indicadores precisa ser revisado continuamente, com a inclusão de novos indicadores, retirada de outros ou mesmo reformulações para atender às expectativas do gerenciamento da drenagem urbana (São Paulo, 2012). Essa possível descontinuidade na coleta de informações não permite a avaliação de desempenho do sistema ao longo dos anos, dificultando a avaliação de políticas existentes e a formulação de novas.

Por fim, destaca-se a importância da publicidade dos indicadores e de seus resultados que, além de informar a condição de determinada atividade ou processo, tornam a gestão transparente e permitem que a sociedade possa contribuir. Com exceção dos indicadores do SNIS, são raros os casos dos municípios que divulgam os resultados de seus indicadores referentes ao serviço de águas pluviais.

\section{Aspectos legais}

No Brasil, os municípios são os responsáveis pelo planejamento dos serviços de drenagem e manejo de águas pluviais urbanas, sendo este realizado em escala municipal (Brasil, 2007). No entanto, como é sabido, a drenagem natural das águas pluviais ocorre em escala de bacia hidrográfica e, raramente, os limites dos municípios seguem os limites hidrográficos, o que gera impasses na gestão da drenagem das águas pluviais urbanas, principalmente em regiões em que as áreas urbanas de diferentes municípios pertencem a uma mesma bacia hidrográfica. Como alternativa, a recente Lei Federal n¹4.026/2020 admite a formalização de consórcios intermunicipais de saneamento básico, exclusivamente compostos por municípios, que poderão prestar o serviço aos seus consorciados diretamente, pela instituição de autarquia intermunicipal, além do estabelecimento de blocos de referência, que permitirá a prestação regionalizada dos serviços de saneamento (Brasil, 2020). Essa legislação ainda induz o município a participar dos blocos regionais, uma vez que o ente municipal que não aderir ao bloco regional não terá prioridade na captação de investimentos federais. Ademais, a prestação regionalizada permite que haja maior compatibilidade entre as ações realizadas no serviço de drenagem de águas pluviais urbanas em diferentes municípios pertencentes a uma mesma bacia hidrográfica, desde que pertençam a um mesmo bloco.

Rasch (2016) assegura que a má governança, caracterizada por assentamentos informais e favelas, é um problema que assola todas as regiões do Brasil, mesmo em municípios com níveis relativamente altos de status socioeconômico. Até 2020 não havia uma agência nacional responsável pelas normas de referências para a área de saneamento no Brasil. A Lei Federal n 14.026/2020 delega à Agência Nacional das Águas (ANA) essas atribuições (Brasil, 2020), sendo que é esperada uma padronização de abrangência nacional e o estabelecimento de parâmetros mínimos a serem observados. A ausência de uma base de dados confiáveis relacionados às águas pluviais é um dos maiores gargalos na formulação de ferramentas baseadas em indicadores. Moura et al. (2009), durante o desenvolvimento do conjunto de indicadores para a avaliação dos efeitos da urbanização nos corpos de água, destacam não só a indisponibilidade efetiva de informações, como também a dificuldade de coleta dados (quando existentes) uma vez que se encontram, normalmente, organizados de forma precária. Esse gargalo na formulação de ferramentas baseadas em indicadores pode induzir os formuladores a inferências, simplificações e estimativas que prejudicam a qualidade e a confiabilidade dos resultados (Moura et al., 2009)

Além da utilização dos resultados dos indicadores pelos gestores públicos para direcionar investimentos e compreender o sistema de águas pluviais urbanas, estes devem ser mais explorados para validar o desenvolvimento de políticas públicas. Devido ao elevado custo de muitas das obras e de muitas não serem visíveis ou utilizadas ininterruptamente, apesar da sua reconhecida importância, esse tipo de infraestrutura é negligenciada (Nickel, 2018). Marlow et al. (2013) destacam que pressões associadas às mudanças climáticas e mudanças nas populações e demografias têm potencial para mudar essa situação e haver reconhecimento da importância da infraestrutura de drenagem das águas pluviais urbanas.

\section{CONCLUSÃo}

A avaliação dos conjuntos de indicadores e índices relacionados ao sistema de águas pluviais desenvolvidos para a realidade brasileira e identificação os entraves existentes e as oportunidades de 
evolução, realizadas com base em levantamento bibliográfico e documental, permitiram a indicação de considerações para a evolução dos mesmos, as quais seguem:

- Indicadores de processo, com um menor nível de agregação, podem ser mais interessantes para o gerenciamento das águas pluviais nos municípios brasileiros atualmente, por facilitarem a compreensão dos principais problemas existentes, assim como já vem sendo aplicados, majoritariamente, nos Planos Municipais de Saneamento, cabendo esforços para evolução dos mesmos.

- Os dados, a quantidade de indicadores e os pesos dos indicadores variam de acordo com o objetivo que se pretende alcançar com determinado conjunto de indicadores/índice. Para fins municipais é fundamental que os dados que as compõem sejam de fácil obtenção e que o conjunto de indicadores/índice sejam de fácil manuseio, sem deixar, porém, de atender ao objetivo.

- Indicadores/índices qualitativos, desde que normalizados e ponderados de forma adequada a realidade do local onde serão utilizados e a finalidade a qual se destinam, são úteis por permitirem acompanhar a evolução ao longo do tempo de determinado aspecto ou processo.

- Para além da área municipal como espaço de planejamento, é importante a observância da espaço da bacia hidrográfica para fins de planejamento de ações, principalmente em regiões intensamente urbanizadas, como regiões metropolitanas, onde a área urbana de mais de um município pode abranger uma mesma bacia hidrográfica.

- $\quad$ É de suma importância que haja padronização de abrangência nacional de normas de referência e o estabelecimento de parâmetros mínimos a serem observados para o serviço de drenagem das águas urbanas pluviais, de forma a permitir a evolução que políticas públicas, além do direcionamento adequado de investimentos no setor. Os conjuntos de indicadores e índices analisados demostraram ser possível construirmos e utilizarmos indicadores que contemples as áreas temáticas, conforme estipulado atualmente no ordenamento jurídico.

- Algumas recomendações são indicadas quando da formulação de indicadores para fins de suporte à gestão de águas pluviais urbanas, como a consideração e indicação das incertezas associadas ao processo de desenvolvimento dos indicadores/índices, consideração de fatores climáticos e que sejam compreensíveis aos diversos atores envolvidos.

\section{AGRADECIMENTOS}

A primeira autora agradece a concessão da bolsa de estudos pela agência brasileira Coordenação de Aperfeiçoamento de Pessoal de Nível Superior.

\section{REFERÊNCIAS}

Araújo, D. C., Silva, P. O., Curi, W. F., \& Cabral, J. J. S. P. (2017). Multicriteria analysis applied to the management of urban pluvial waters. Brazilian Journal of Water Resources, 22(0), http://dx.doi.org/10.1590/23180331.011716006

Baptista, M., Barraud, S., Alfakih, E., Nascimento, N., Fernandes, W., Moura, P., \& Castro, L. (2005). Performancecosts evaluation for urban storm drainage. Water Science and Technology, 51(2), 99-107. http://dx.doi.org/10.2166/wst.2005.0037

Barbosa, A. E., Fernandes, J. N., \& David, L. M. (2012). Key issues for sustainable urban stormwater management. Water Research, 46(20), 6787-6798. http://dx.doi.org/10.1016/j.watres.2012.05.029

Barroca, B., Bernardara, P., Mouchel, J. M., \& Hubert, G. (2006). Indicators for identification of urban flooding vulnerability. Natural Hazards and Earth System Sciences, 6(4), 553-561. http://dx.doi.org/10.5194/nhess6-553-2006

Bauler, T. (2012). An analytical framework to discuss the usability of (environmental) indicators for policy. Ecological Indicators, 17, 38-45. http://dx.doi.org/10.1016/j.ecolind.2011.05.013

Belém. (2020). Plano Municipal de Saneamento Básico - Belém/PA. Recuperado em 1 de outubro de 2020, de http://www.belem.pa.gov.br/arbel/?page_id=723

Belo Horizonte. (2020). Plano Municipal de Saneamento de Belo Horizonte 2020/2023. Recuperado em 1 de outubro de 2020, de https://prefeitura.pbh.gov.br/obras-e-

infraestrutura/informacoes/publicacoes/plano-de-saneamento 
Bertilsson, L., Wiklund, K., de Moura Tebaldi, I., Rezende, O. M., Veról, A. P., \& Miguez, M. G. (2019). Urban flood resilience: a multi-criteria index to integrate flood resilience into urban planning. Journal of Hydrology, 573, 970-982. http://dx.doi.org/10.1016/j.jhydrol.2018.06.052

Bertrand-Krajewski, J.-L. (2021). Integrated urban stormwater management: evolution and multidisciplinary perspective. Journal of Hydro-environment Research, 38, 72-83. http://dx.doi.org/10.1016/j.jher.2020.11.003

Birgani, Y. T., \& Yazdandoost, F. (2014). A framework for evaluating the persistence of urban drainage risk management systems. Journal of Hydro-environment Research, 8(4), 330-342. http://dx.doi.org/10.1016/j.jher.2014.04.004

Brasil. (2007). Lei no 11.445, de 5 de janeiro de 2007. Diário Oficial [da] República Federativa do Brasil, Brasília. Recuperado em 15 de maio de 2021, de http://www.planalto.gov.br/ccivil_03/_ato20072010/2007/lei/l11445.htm\#: :text=Estabelece\%20diretrizes\%20nacionais\%20para\%20o,1978\%3B\%20 e\%20d\%C3\%A1\%20outras\%20provid\%C3\%AAncias.

Brasil. (2020). Lei no 14.026, de 15 de julho de 2020. Diário Oficial [da] República Federativa do Brasil, Brasília. Recuperado em 15 de maio de 2021, de http://www.planalto.gov.br/ccivil_03/_ato20192022/2020/lei/l14026.htm

Castro, L. M. A., Baptista, M. B., \& Netto, O. M. C. (2004). Análise multicritério para a a evolução dos sistemas de drenagem urbana. Revista Brasileira de Recursos Hídricos, 9(4), 5-19. Recuperado em 15 de maio de 2021, de https://abrh.s3.sa-east1.amazonaws.com/Sumarios/30/6f432d324627eb2288c1efc77f68ce95_3c2ad480b3655705a1b531f3c68c 279f.pdf

Castro, L. M. A., Baptista, M. B., \& Barraud, S. (2009). Proposição de metodologia para a avaliação dos efeitos da urbanização nos corpos de água. Revista Brasileira de Recursos Hídricos, 14(4), 103-112. http://dx.doi.org/10.21168/rbrh.v14n4.p113-123

Cavalcanti Filho, M. J. L. (2017). Desenvolvimento e avaliação de um conjunto de indicadores para representação do sistema de drenagem urbana (Dissertação de mestrado). Centro de Tecnologia, Universidade Federal de Alagoas, Maceió.

Colombelli, K. (2018). Serviço público de drenagem e manejo de águas pluviais urbanas: avaliação do contexto brasileiro e da adaptabilidade de práticas norte-americanas para a proposição de melhorias institucionais e financeiras (Dissertação de mestrado). Universidade Federal do Rio Grande do Sul, Porto Alegre.

Cometti, J. L. S., Cabral, J. J. P. S., \& Conceição, T. M. (2019). Indicadores de pressão-estado-resposta para avaliação da conservação ambiental de riachos urbanos. Revista Ibero-Americana de Ciências Ambientais, 10(6), 194-205. http://dx.doi.org/10.6008/CBPC2179-6858.2019.006.0017

Debortoli, N. S., Camarinha, P. I. M., Marengo, J. A., \& Rodrigues, R. R. (2017). An index of Brazil's vulnerability to expected increases in natural flash flooding and landslide disasters in the context of climate change. Natural Hazards, 86(2), 557-582. http://dx.doi.org/10.1007/s11069-016-2705-2

Distrito Federal. (2017). Plano distrital de saneamento básico do Distrito Federal. Recuperado em 1 de outubro de 2020, de https://www.adasa.df.gov.br/images/ProdutosPDSB/Produto_7/1_PDSB_DF_subproduto_7.1_0717_VF_DIGITAL.pdf

Dizdaroglu, D. (2015). Developing micro-level urban ecosystem indicators for sustainability assessment. Environmental Impact Assessment Review, 54, 119-124. http://dx.doi.org/10.1016/j.eiar.2015.06.004

Fletcher, T. D., Shuster, W., Hunt, W. F., Ashley, R., Butler, D., Arthur, S., Trowsdale, S., Barraud, S., SemadeniDavies, A., Bertrand-Krajewski, J.-L., Mikkelsen, P. S., Rivard, G., Uhl, M., Dagenais, D., \& Viklander, M. (2015) SUDS, LID, BMPs, WSUD and more: the evolution and application of terminology surrounding urban drainage. Urban Water Journal, 12(7), 525-542. http://dx.doi.org/10.1080/1573062X.2014.916314

Hammond, A., Adriaanse, A., Rodenburg, E., Bryant, D., \& Woodward, R. (1995). Environmental indicators: a systematic approach to measuring and reporting on environmental policy performance in the context of sustainable development (Vol. 36). Washington, DC: World Resources Institute. Recuperado em 15 de maio de 2021, de http://pdf.wri.org/environmentalindicators_bw.pdf

Holz, J. (2010). Levantamento e mapeamento do índice de risco de alagamento da bacia do Riacho Reginaldo (Dissertação de mestrado). Centro de Tecnologia, Universidade Federal Alagoas, Maceió.

Huang, L., Wu, J., \& Yan, L. (2015). Defining and measuring urban sustainability: a review of indicators. Landscape Ecology, 30(7), 1175-1193. http://dx.doi.org/10.1007/s10980-015-0208-2 
Igroufa, M., Benzerra, A., \& Seghir, A. (2020). Development of an assessment tool for infrastructure asset management of urban drainage systems. Water Science and Technology, 82(3), 537-548. http://dx.doi.org/10.2166/wst.2020.356

Jia, H., Yao, H., Tang, Y., Yu, S. L., Zhen, J. X., \& Lu, Y. (2013). Development of a multi-criteria index ranking system for urban runoff best management practices (BMPs) selection. Environmental Monitoring and Assessment, 185(9), 7915-7933. http://dx.doi.org/10.1007/s10661-013-3144-0

Kaur, H., \& Garg, P. (2019). Urban sustainability assessment tools: A review. Journal of Cleaner Production, 210, 146-158. http://dx.doi.org/10.1016/j.jclepro.2018.11.009

Klopp, J. M., \& Petretta, D. L. (2017). The urban sustainable development goal: Indicators, complexity and the politics of measuring cities. Cities, 63, 92-97. http://dx.doi.org/10.1016/j.cities.2016.12.019

Kolsky, P., \& Butler, D. (2002). Performance indicators for urban storm drainage in developing countries. Urban Water, 4(2), 137-144. http://dx.doi.org/10.1016/S1462-0758(02)00011-0

Marlow, D. R., Moglia, M., Cook, S., \& Beale, D. J. (2013). Towards sustainable urban water management: a critical reassessment. Water Research, 47(20), 7150-7161. http://dx.doi.org/10.1016/j.watres.2013.07.046

Marques, G. E. B. (2006). Proposta de método para a formulação de planos diretores de drenagem urbana (Dissertação de mestrado). Universidade de Brasília, Brasília.

Martin, C., Ruperd, Y., \& Legret, M. (2007). Urban stormwater drainage management: the development of a multicriteria decision aid approach for best management practices. European Journal of Operational Research, 181(1), 338-349. http://dx.doi.org/10.1016/j.ejor.2006.06.019

Mayer, A. L. (2008). Strengths and weaknesses of common sustainability indices for multidimensional systems. Environment International, 34(2), 277-291. http://dx.doi.org/10.1016/j.envint.2007.09.004

McClymont, K., Fernandes Cunha, D. G., Maidment, C., Ashagre, B., Vasconcelos, A. F., Batalini de Macedo, M., Nóbrega dos Santos, M. F., Gomes Júnior, M. N., Mendiondo, E. M., Barbassa, A. P., Rajendran, L., \& Imani, M. (2020). Towards urban resilience through Sustainable Drainage Systems: a multi-objective optimisation problem. Journal of Environmental Management, 275, 111173. http://dx.doi.org/10.1016/j.jenvman.2020.111173

McGrane, S. J. (2016). Impacts of urbanisation on hydrological and water quality dynamics, and urban water management: a review. Hydrological Sciences Journal, 61(13), 2295-2311. http://dx.doi.org/10.1080/02626667.2015.1128084

Mendonça, E. C., \& Souza, M. A. A. (2019). Uma metodologia multiobjetivo e multicritério para avaliação de desempenho de sistemas de drenagem urbana. Ingeniería Del Agua, 23(2), 89. http://dx.doi.org/10.4995/ia.2019.10214

Miguez, M. G., \& Veról, A. P. (2017). A catchment scale Integrated Flood Resilience Index to support decision making in urban flood control design. Environment and Planning B: Urban Analytics and City Science, 44(5), 925-946. http://dx.doi.org/10.1177/0265813516655799

Miranda, F. M., Sousa, M. M., Rezende, M., Caroline, A., Jacob, P., \& Gomes, M. (2019). Simplified flood risk index applied to the Duque de Caxias Municipality in the Metropolitan Region of Rio de Janeiro. NOVATECH, 1-4. Recuperado em 15 de maio de 2021, de http://www.novatech.graie.org/documents/auteurs/3B9P207MIR.pdf

Morales-Torres, A., Escuder-Bueno, I., Andrés-Doménech, I., \& Perales-Momparler, S. (2016). Decision Support Tool for energy-efficient, sustainable and integrated urban stormwater management. Environmental Modelling \& Software, 84, 518-528. http://dx.doi.org/10.1016/j.envsoft.2016.07.019

Moura, P. M., Baptista, M. B., \& Barraud, S. (2009). Avaliação multicritério de sistemas de drenagem urbana. Revista de Gestão de Água da América Latina, 6(1), 31-42. Recuperado em 15 de maio de 2021, de https://www.abrhidro.org.br/SGCv3/publicacao.php?PUB=2\&ID=73\&SUMARIO=866

Moura, P. M., Barraud, S., \& Baptista, M. B. (2010). Metodologia para avaliação de sistemas de infiltração de águas pluviais urbanas-fase de concepção. Revista de Gestão de Água da América Latina, 7(2), 5-16. http://dx.doi.org/10.21168/rega.v7n2.p5-16

Natal. (2015). Plano Municipal de Saneamento Básico de Natal/RN. Recuperado em 1 de outubro de 2021, de htts://www2.natal.rn.gov.br > seharpe > File > PROGNOSTICO_PMSB_NATAL.pdf

Nickel, D. (2018). Water in the city of the future. Urban Water Journal, 15(6), 507. http://dx.doi.org/10.1080/1573062X.2018.1528729 
Oneda, T. M. S. (2018). Planos Diretores de Drenagem Urbana: uma análise comparativa entre planos de países desenvolvidos e em desenvolvimento (Dissertação de mestrado). Universidade do Estado de Santa Catarina, Joinville.

Pereira, C., Miguez, M., Di Gregório, L., Haddad, A., \& Vérol, A. (2020). Inundation risk index as an urban planning supportive tool. Journal of Sustainable Development of Energy. Water and Environment Systems, 8(2), 235251. http://dx.doi.org/10.13044/j.sdewes.d7.0288

Perez, L. P. (2013). Índice de vulnerabilidade urbana a alagamentos e deslizamentos de terra, em função de eventos extremos de clima, na Região Metropolitana de São Paulo: uma proposta de método (Tese de doutorado). Faculdade de Filosofia, letras e Ciências Humanas, Universidade de São Paulo, São Paulo. http://dx.doi.org/10.11606/T.8.2013.tde-12032014-121536

Porto Alegre. (2015). Plano Municipal de Saneamento de Porto Alegre/RS. Recuperado em 1 de outubro de 2021, de https://www2.portoalegre.rs.gov.br/dmae/default.php?p_secao=352

Rasch, R. J. (2016). Assessing urban vulnerability to flood hazard in Brazilian municipalities. Environment and Urbanization, 28(1), 145-168. http://dx.doi.org/10.1177/0956247815620961

Rezende, O. M., Cruz de Franco, A. R., Beleño de Oliveira, A. K., Jacob, A. C. P., \& Miguez, M. (2019). A framework to introduce urban flood resilience into the design of flood control alternatives. Journal of Hydrology, 576, 478-493. http://dx.doi.org/10.1016/j.jhydrol.2019.06.063

Rezende, O. M., Ribeiro da Cruz de Franco, A. B., Beleño de Oliveira, A. K., Miranda, F. M., Pitzer Jacob, A. C., Martins de Sousa, M., \& Miguez, M. G. (2020). Mapping the flood risk to Socioeconomic Recovery Capacity through a multicriteria index. Journal of Cleaner Production, 255, 120251. http://dx.doi.org/10.1016/j.jclepro.2020.120251

Rio Grande do Sul. (2021). Plano Estadual de Saneamento (PLANESAN-RS): relatório de Porto Alegre. Porto Alegre. Recuperado em 1 de outubro de 2021, de https://www.sema.rs.gov.br/relatorio-de-salubridadeambiental

Ripol e Silva, B., Pinheiro, H., \& Lopes, D. D. (2013). Avaliação do sistema de drenagem urbana da cidade de Vitória - ES. Revista Nacional de Gerenciamento de Cidades, 1(1), 30-44.

Romnée, A., Evrard, A., \& Trachte, S. (2015). Methodology for a stormwater sensitive urban watershed design. Journal of Hydrology, 530, 87-102. http://dx.doi.org/10.1016/j.jhydrol.2015.09.054

Rotava, J. (2014). Índices de resiliência hídrica e de perigo para gestão do risco de inundação urbanas (Dissertação de mestrado). Universidade de São Paulo, São Carlos.

Santos Júnior, V. J. (2014). Avaliação da fragilidade no sistema de drenagem pluvial urbana: o caso da bacia hidrográfica do Córrego das Melancias em Montes Claros - MG. Revista Monografias Ambientais, 13(5), 3986-3997. http://dx.doi.org/10.5902/2236130815125

São Paulo. (2012). Manual de drenagem e manejo de águas pluviais. Secretaria Municipal de Desenvolvimento urbano. Recuperado em 15 de maio de 2021, de https://www.prefeitura.sp.gov.br/cidade/secretarias/upload/desenvolvimento_urbano/arquivos/manualdrenagem_v1.pdf

Sepe, P. M. I., \& Gomes, S. (2008). Indicadores ambientais e gestão urbana desafi os para a construção da sustentabilidade na cidade de São Paulo. São Paulo: Centro de Estudos da Metrópole, Secretaria Municipal do Verde e do Meio Ambiente. Recuperado em 15 de maio de 2021, de http://web.fflch.usp.br/centrodametropole/antigo/v1/pdf_2009/LivroMeioAmbiente.pdf

Shields, D. J., Šolar, S. V., \& Martin, W. E. (2002). The role of values and objectives in communicating indicators of sustainability. Ecological Indicators, 2(1-2), 149-160. http://dx.doi.org/10.1016/S1470-160X(02)00042-0

Silva de Brito, D. (2006). Metodologia para seleção de alternativas de sistemas de drenagem (Dissertação de mestrado). Universidade de Brasília, Brasília.

Silva, S. (2016). Ferramenta de apoio ao manejo de águas pluviais urbanas com base em indicadores de sustentabilidade - SAMSAP (Tese de doutorado). Universidade Federal de São Carlos, São Carlos. https://doi.org/10.1063/1.1726707.

Sistema Nacional de Informação sobre Saneamento-Águas Pluviais - SNIS-AP. (2019). 3o diagnóstico de drenagem e manejo das águas pluviais urbanas - 2018. Brasília: MCIDADES.SNSA. Recuperado em 15 de maio de 2021, de http://www.snis.gov.br/diagnostico-anual-aguas-pluviais/diagnostico-do-servico-deaguas-pluviais-2018

Streimikiene, D., \& Šivickas, G. (2008). The EU sustainable energy policy indicators framework. Environment International, 34(8), 1227-1240. http://dx.doi.org/10.1016/j.envint.2008.04.008 
Szlafsztein, C., \& Sterr, H. (2007). A GIS-based vulnerability assessment of coastal natural hazards, state of Pará, Brazil. Journal of Coastal Conservation, 11(1), 53-66. http://dx.doi.org/10.1007/s11852-007-0003-6

Teixeira, D. A., Prado Filho, J. F., \& Santiago, A. F. (2018). Indicator of environmental health: formula modifications and indicator applications in Brazil. Engenharia Sanitária e Ambiental, 23(3), 543-556. http://dx.doi.org/10.1590/s1413-41522018170866

van Bellen, H. M. (2005). Indicadores de sustentabilidade: uma análise comparativa (256 p.). Rio de Janeiro: FGV Editora.

Vasconcelos, A., \& Barbassa, A. (2021). Strategies to overcome barriers and to effectively achieve sustainable urban stormwater management in developing countries. Urban Water Journal. In press. http://dx.doi.org/10.1080/1573062X.2021.1969415

Verma, P., \& Raghubanshi, A. S. (2018). Urban sustainability indicators: challenges and opportunities. Ecological Indicators, 93, 282-291. http://dx.doi.org/10.1016/j.ecolind.2018.05.007

Veról, A. P., Battemarco, B. P., Merlo, M. L., Machado, A. C. M., Haddad, A. N., \& Miguez, M. G. (2019). The urban river restoration index (URRIX): a supportive tool to assess fluvial environment improvement in urban flood control projects. Journal of Cleaner Production, 239, 118058. http://dx.doi.org/10.1016/j.jclepro.2019.118058

von Ancken, N. B., Teixeira, B. A. N., \& Silva, S. P. (2017). Aplicação de indicadores de sustentabilidade para o manejo de águas pluviais: estudo de caso em São Carlos-SP. Florianópolis: ABRH.

Zonensein, J. (2007). Índice de risco de cheia como ferramenta de gestão de enchentes (Dissertação de mestrado). Universidade Federal do Rio de Janeiro, Rio de Janeiro.

\section{Contribuições dos autores:}

Camila Angélica Baum: realizou as atividades de construção científica do artigo, busca de dados e referências, análise e discussão dos resultados e redação do artigo.

Joel Avruch Goldenfum: participou da construção científica do artigo, orientou os resultados e revisou o manuscrito do texto. 